\title{
Seguridad humana en Soacha desde la percepción local. Análisis del impacto de los proyectos de la FAO y el PNUD ${ }^{1}$
}

\section{Human Security in Soacha through the local perception. Analysis of the impact of FAO's and UNDP's projects}

Enrico Nichelatti ${ }^{2}$

Fecha de recepción: 01/07/2020 - Fecha de aceptación: 19/11/2020

DOI: https://doi.org/10.22490/26655489.4109

\section{Resumen}

El artículo parte de la concepción de la seguridad humana, que resume, y analiza proyectos desarrollados en Soacha por dos organismos especializados de NN. UU., FAO y PNUD. El caso de Soacha ha sido elegido por representar un contexto único. La comuna de Soacha vive una paradoja por la cual reúne en un mismo lugar los problemas históricos y emergentes de Colombia. La precariedad asociada a las zonas rurales del país (desigualdad, concentración de la tierra, extrema pobreza, desplazamientos forzados) se junta en Soacha con la mirada puesta en el futuro que representa la colindante Bogotá. El estudio usa un enfoque cualitativo que se articula en dos partes. Por un lado, se llevan a cabo entrevistas personales semiestructuradas con los coordinadores de los proyectos, para averiguar cuáles son los obstáculos que los proyectos enfrentan en su implementación, tanto a corto como a largo plazo. Por otro lado, se realizan encuestas a 120 personas, consistentes en un preámbulo y ocho preguntas, en las cuales se recopila información sobre la percepción de la población sobre el estado de la seguridad humana en su territorio. Los resultados muestran que la población considera que se ha producido una ligera mejora en la seguridad

1 Artículo de investigación científica.

2 Máster en "International Cooperation and Development" y actualmente cursando un máster en "Public Policy and Human Developement" en la Universidad de Naciones Unidas/Universidad de Maastricht. Correo electrónico: nichelatti93@gmail.com ORCID ID: https://orcid.org/0000-0002-1026-2675 
humana en los últimos cinco años. Sin embargo, los resultados también evidencian la persistencia de muchos de los problemas estructurales que perpetúan la inseguridad humana.

Palabras clave: seguridad humana, Soacha, Naciones Unidas, FAO, PNUD.

\section{Abstract}

The research wants to focus on the concept of human security through a qualitative analysis of the impact of some projects developed by the Food and Agriculture Organization and United Nations Developemnt Pogramme in Soacha. The place represents an interesting case study for its particular situation. Indeed, this commune on the one hand still lives historical issues such as extreme poverty, inequality, accumulation of land and displacement, and on the other hand represents a paradoxical union between precarious and developed realities. The study uses a qualitative technique divided in two sections. The first one is built on semi structured individual interviews with the project's coordinators that examine the main issues with their implementation, while the other one is based on surveys with the local community that analyze the perception of the population of Soacha on human security. Regarding this part, the survey, composed by a preamble and eight questions, has been completed by 120 people. The results evidence that the population consider that there has been a slight increase in the human security during the last five years. However, the outcomes also reveal the persistence of structural issues, which perpetuate the human insecurity.

Keywords: Human security, Soacha, United Nations, FAO, UNDP.

\section{Seguridad humana}

La seguridad humana es un concepto profundamente innovador si se compara con el concepto tradicional de seguridad, centrado exclusivamente en la seguridad militar -a veces también política- de los Estados, que ofrece un enfoque amplio y multidimensional, basado en siete dimensiones altamente interrelacionadas entre ellas (al igual que las amenazas y retos): 
a. Seguridad económica frente a amenazas como la pobreza persistente o el desempleo.

b. Seguridad alimentaria frente a hambrunas y escasez de alimentos.

c. Seguridad sanitaria frente a enfermedades infecciosas, alimentación insegura, desnutrición y la falta de acceso al sistema de salud.

d. Seguridad medioambiental frente a la degradación del ecosistema, el agotamiento de recursos, los desastres naturales, la contaminación y el cambio climático.

e. Seguridad personal frente a la violencia física, los crímenes y delitos, el terrorismo y la violencia doméstica y de género.

f. Seguridad comunitaria frente a las tensiones interétnicas y religiosas, entre otras.

g. Seguridad política frente a la represión o a la violación de derechos humanos (United Nations Development Programme, 1994).

Garantizar o conseguir la seguridad humana en estas siete dimensiones implica llevar a cabo dos estrategias: la protección y el empoderamiento. La primera implica establecer medidas descendentes, reconociendo que las personas se enfrentan a amenazas que no están bajo su control; por esto, es fundamental la protección sistemática, integral y preventiva de las comunidades humanas. Los Estados son los principales responsables de proveer este tipo de protección, pero deben colaborar con otros actores en distintos niveles, como los organismos internacionales, la sociedad civil y las organizaciones no gubernamentales (Inte-American Institute of Human Rights \& United Nations Development Programme, 2012). El empoderamiento, por su parte, es una estrategia que se basa sobre el concepto de crear y desarrollar capacidades de resiliencia en las personas y en las comunidades humanas en su conjunto. El objetivo de esta estrategia ascendente es permitir que personas y comunidades puedan anticipar las posibles amenazas y así puedan evitarlas o resistirlas, e intentar limitar sus daños en el caso de que sean inevitables. Diseñar una política de seguridad humana implica planificar una actuación articulada en tres fases. En primer lugar, hay que trazar el mapa de las necesidades y vulnerabilidades de las personas. Este aspecto negativo debe ir seguido del análisis positivo de las capacidades de las comunidades interesadas. 
El diseño de las actuaciones en el terreno sigue los principios de:

a. Centrarse en las personas

b. Adoptar un enfoque multisectorial, que abarque todas las dimensiones de la seguridad humana afectadas y que establezca la coordinación y sinergia entre todos los actores involucrados.

c. Adaptarse a las peculiaridades de cada contexto sociopolítico, cultural y económico.

d. Actuar siempre bajo el principio de prevención orientada hacia las personas, intentando minimizar las posibles. consecuencias personales negativas que podrían presentarse en un futuro (Office for the Coordination of Humanitarian Affairs, 2009).

La segunda fase consiste en la implementación de la política, con el objetivo de garantizar la participación de los destinatarios de las políticas y los otros actores con capacidad de movilización de recursos en el contexto local, provincial o departamental. Para hacer esto, la política debe garantizar el desarrollo de capacidades de las comunidades involucradas y monitorear su grado de asociación y colaboración. De hecho, un programa de seguridad humana sólido debe evaluarse en función al nivel de participación local en la fase de implementación y sostenibilidad del programa. (Office for the Coordination of Humanitarian Affairs. 2009).

La tercera y última fase corresponde a la evaluación del impacto, que puede tomar distintas formas y tener objetivos muy variados. Casi siempre se piensa realizar la evaluación al final de un programa o después de la implementación para evaluar su éxito. Pero la evaluación también puede llevarse a cabo durante el ciclo de vida del programa para estimar el progreso y, cuando sea necesario, plantear cambios para mejorar o redirigir el programa. Desde una perspectiva de seguridad humana, la evaluación debe centrarse en la evaluación del impacto (Office for the Coordination of Humanitarian Affairs, 2009). 


\section{Soacha}

En toda la región de Latinoamérica, a nivel poblacional, no hay ningún lugar que haya crecido con un nivel más alto que Soacha, llegando hasta el $12 \%$ en la década de los 80 (EI Tiempo, 1999). Hay mucha incertidumbre y especulaciones sobre el número efectivo de ciudadanos residentes en este municipio. Mientras la Secretaría de Planeación Municipal calcula que viven 700 mil personas, la Gobernación de Cundinamarca afirma que hay 1,1 millones y los líderes de Altos de la Cazucá llegan hasta 1,5 millones (El Tiempo, 1999). El crecimiento de la población de Soacha es una consecuencia clara del fenómeno de las migraciones internas, un desafío importante para toda Colombia. En 1938 , el 30,9 \% de la población nacional residía en las áreas urbanas, en 1951 el 39,6 \% y en 1964 el 52,1\%. En 1973, esta cifra se eleva hasta el 63,1\%, para llegar al 67,2 \% en 1985 y al $72,3 \%$ en 1994. La migración masiva, rápida y descontrolada desde las áreas agrícolas a las urbanas tiene un fuerte impacto negativo en la prestación de servicios públicos y en la satisfacción de las necesidades básicas (Niño Pavajeau, 1999).

Conflictos armados, eventos de origen natural, desplazamientos forzados, desigualdad en la propriedad de la tierra, junto a la fuerte presencia de comunidades vulnerables expuestas a la extrema pobreza, siguen siendo las principales causas de este fenómeno. A este escenario se añade la compleja situación política y económica de Venezuela, que obliga cada día a miles de sus ciudadanos a escapar a Colombia. Estos dos aspectos provocan que las Comunas como Soacha, por sus posiciones estratégicas, crezcan sin control. Como demostración de eso, el $80 \%$ de sus habitantes duermen en esa zona, pero trabajan o estudian en Bogotá D. C. (Castellanos, 1991). La provincia de Soacha se caracteriza por tener un alto nivel de vulnerabilidad de su población: su municipio recibe una gran cantidad de personas que emigran desde otros municipios del país en busca de mejores oportunidades de vida (Chamber of Commerce of Bogota, 2012) o que viven una situación de desplazamiento (PSD).

Hay otros dos factores con importantes efectos para el difícil equilibrio de esta zona. Gran parte de la población es víctima de la necesidad de vivienda y de los traficantes de tierras o urbanizadores piratas (Castellanos, 1991). En relación con este aspecto, tómese como ejemplo el barrio Altos de Florida, construido 
aproximadamente hace 30 años por personas desplazadas que buscaban construir su propio refugio o por personas que no tenían una condición socioeconómica suficiente para pagar un arriendo. En este barrio no hay colegios ni servicio de transporte público, así que los jóvenes están obligados a ir caminando a las escuelas. Esta situación expone los jóvenes a los peligros de la zona, provocando una alta tasa de abandono escolar. Entre los otros problemas más relevantes de Altos de Florida, hay que mencionar la tragedia del agua, que llega solo en carrotanque, el altísimo nivel de inseguridad y violencia, la falta de transporte y el alto nivel de contaminación, debido a la violación de las normas medioambientales (Gallego Suárez, 2011).

A pesar del tamaño considerable de Soacha, la capacidad institucional del Gobierno municipal en términos administrativos y operativos es extremadamente débil (Chamber of Commerce of Bogota, 2005), por el hecho de que no ha podido desarrollarse al mismo ritmo que la población y tiene serios problemas de inestabilidad política. El informe de la Cámara de Comercio de Bogotá señala, además, que esta inestabilidad política provoca la falta de transparencia del sector público y la falta de coordinación entre los sectores público y privado, junto a la falta de una cultura de planificación a medio y largo plazo (Dávila, 2013).

Las amenazas por mano de los grupos armados y organizados son la causa principal del desplazamiento inter e intraurbano. En particular para la población infantil y juvenil, el reclutamiento forzado es una amenaza directa muy preocupante (United Nations High Commissioner for Refugees, 2013). Los grupos armados presentes en el municipio tienen militantes anteriormente pertenecientes a los grupos guerrilleros FARC y ELN, mientras que las organizaciones criminales están conectadas con los carteles mexicanos del narcotráfico. Este factor contribuye a la inseguridad de esta zona mediante homicidios, secuestros, amenazas, venta de droga y conflictos internos (Ocampo Rodríguez, 2019).

Soacha tiene una única y peligrosa combinación entre la presencia de comunidades vulnerables, altos niveles de desigualdad y pobreza extrema, altos niveles de corrupción, violencia, ilegalidad y grave falta de las instituciones. Esa combinación hace que ese municipio sea uno de los más complejos por su situación actual y su frágil equilibrio. 


\section{Análisis de los proyectos}

El trabajo pone particular atención en el análisis de algunos proyectos desarrollados en el municipio de Soacha. Los criterios con los cuales han sido elegidos los proyectos son tres: su grado de correlación con al menos una dimensión de la seguridad humana, el tamaño del proyecto y la organización a cargo del proyecto. La elección final recae en seis proyectos: tres de ellos son llevados a cabo por la FAO. Uno es sobre la construcción de huertas escolares, otro sobre el fortalecimiento de la seguridad alimentaria en cooperación con el proyecto "Familias en Acción", y el último sobre la cultivación urbana. El cuarto proyecto elegido es implementado por el PNUD, en colaboración con otras organizaciones del sistema ONU, como el Alto Comisionado para los Refugiados (ACNUR). El quinto y el sexto proyecto son el fruto de la colaboración entre la organización "Prosperidad Social" y las Alcaldías de Bogotá y Soacha. Esta elección permite comparar cualitativamente los resultados de proyectos implementados por organismos internacionales pertenecientes a la red de las Naciones Unidas con proyectos de ámbito nacional y local (el quinto y el sexto).

Para profundizar en el análisis de los resultados de estos proyectos, el día 10 de junio de 2019 se ha procedido a entrevistar a los coordinadores de los proyectos o del personal implicado. Gracias a las entrevistas con las señoras María Consuelo Vergara Holguín (asistente de programas de gestión de riesgo y rehabilitación agropecuaria) y Michela Espinosa (especialista senior en alimentación contra la malnutrición), el estudio incluye información esencial sobre los ámbitos de actuación y las estructuras de los proyectos. Además, la posibilidad de poder discutir y enfrentarme directamente con los coordinadores del proyecto permitió obtener una visión más amplia del contexto, de los principales problemas enfrentados en la fase inicial y en el desarrollo del trabajo, descubriendo los roles tenidos por cada actor implicado. Esta parte es considerada fundamental también para poder analizar luego la percepción de los habitantes sobre la seguridad, estudiando los proyectos desde el punto de vista de los creadores y coordinadores y de las personas involucradas o que viven en el municipio. De hecho, todos los datos sobre estos proyectos que se detallan a continuación han sido proporcionados por los trabajadores entrevistados. 
Los proyectos tienen objetivos sinérgicos, entre ellos: la construcción de huertas escolares, familiares y entrenamiento en la seguridad alimentaria para las familias en la Comuna 4 de Soacha, así como aumentar el nivel de la seguridad humana urbana a través la construcción de centros demostrativos. Asimismo, la FAO y su personal garantizan en cada momento el acceso a los documentos de los proyectos, manteniendo una perfecta transparencia y cooperación, elementos indispensables para el desarrollo de este trabajo.

A continuación, se detallan algunos aspectos de los proyectos relativos a la seguridad humana llevados a cabo por la FAO:

a. La construcción de las huertas escolares tiene como objetivo la mejora del nivel de seguridad alimentaria de los niños, niñas y jóvenes de Soacha, a través de la enseñanza sobre cómo obtener diferentes alimentos (frutas, verduras y legumbres) (González, 2015). El proyecto, empezado en el 2015, involucra jóvenes entre los cinco y los 19 años, y se desarrolla en 14 centros educativos del municipio. El programa prevé también la participación de los adultos en la creación y sostenibilidad de las huertas. La estrategia del proyecto combina el aspecto teórico con el aspecto práctico, convirtiendo la huerta en un instrumento práctico para el aprendizaje de las asignaturas como ciencias naturales, matemáticas y ciudadanía (González, 2015). Gracias al desarrollo de este proyecto, hoy en día, 800 estudiantes producen una considerable parte de sus alimentos en las huertas escolares. Además, para el secretario de educación de Soacha, Abel Suárez, la iniciativa sobrepasa los resultados esperados. De hecho, los estudiantes han aprendido técnicas de producción de alimentos sanos y su incorporación en la dieta y en la prevención de enfermedades generadas por una mala alimentación (González, 2016).

b. El proyecto desarrollado en la Comuna 4 en cooperación con "Familias en Acción" se propone come objetivo fortalecer varias dimensiones de la seguridad alimentaria y favorecer el autoconsumo de productos alimentarios en la comunidad. El programa ha conseguido la instalación de cinco centros demostrativos comunitarios que 
representan un espacio de encuentro para las familias para crear lazos de confianza y para el aprendizaje de diferentes técnicas de agricultura urbana. Esto permite la creación de huertas familiares y, a través de las nuevas técnicas y conocimientos aprendidos sobre la producción y conservación de algunos alimentos, ha permitido el surgimiento de pequeños puntos familiares de venta y puntos de venta móviles gracias a los excedentes productivos. Con todo ello, se han llegado a diseñar y establecer acuerdos para el desarrollo de un sistema de alimentación escolar local a través de la producción local (Rocío \& García, 2007).

c. Un proyecto similar para la agricultura urbana es implementado en el barrio de Altos de Florida que, como se ha dicho anteriormente, surge como área de asentamientos no reconocidos inicialmente por las autoridades. La fase inicial del proyecto se basa sobre un estudio del mercado local para identificar nuevos canales de comercialización y permitir la activación y el surgimiento de una nueva economía local.

Gracias a las entrevistas con las coordinadoras del proyecto, las señoras María Consuelo Vergara Holguín y Olga Rocío Niño García, se pone en evidencia la importancia del componente escolar: uno de los resultados más relevantes consiste en el establecimiento de la cooperación entre los nuevos agricultores locales y los operadores encargados de la venta de productos alimentarios a los comedores escolares. Así, durante esta fase del proyecto, las escuelas se benefician de talleres ofrecidos al personal escolar sobre cómo cocinar y conservar los alimentos, con la supervisión en cada cocina de un técnico de la alimentación. El resultado conseguido es una alianza entra las familias y las escuelas para juntar la comida producida y ponerla en puntos de ventas, garantizando su autonomía. Para las mujeres, esta fase es fundamental porque representa un nuevo ingreso económico considerable y ahora pueden comprar lo que no producen en casa, garantizando una alimentación variada y suficiente en sus hogares. 
Los resultados concretos pueden verse en las siguientes cifras:

1. 14 centros educativos con modelos técnicos concertados para la producción diversificada de alimentos alrededor de las huertas escolares.

2. 500 estudiantes capacitados en técnicas relacionadas con la producción rápida y diversificada de alimentos inocuos.

3. 14 centros han sido provistos de bienes de insumos agrícolas y establecen huertas escolares.

4. 100 padres de familia son capacitados en técnicas para la producción, manipulación, transformación y conservación de alimentos.

5. 32 docentes vinculados a procesos de capacitación en técnicas de producción agroecológica de alimentos.

6. 15 ecónomas capacitadas en manipulación, transformación y conservación de alimentos.

7. 14 comités de huertas escolares conformados para garantizar la sostenibilidad del proceso.

8. 14 centros de producción de semillas y material vegetal conformados (Food and Agriculture Organization, 2015).

Además, las entrevistas con el personal de la FAO permiten evidenciar los problemas más comunes a los varios proyectos implementados: las condiciones precarias del suelo, que está sujeto a degradación y erosión; también, la falta de agua potable y la temperatura alta con las radiaciones solares, el viento fuerte y las precipitaciones. Todos esos representan rasgos locales que dificultan el cultivo. Otro gran problema que se presenta en esta zona es conseguir el acercamiento de la gente de la comuna al proyecto interesado. El alto nivel de desplazados, es decir personas obligadas a dejar sus hogares por los conflictos internos, requiere un proceso de construcción de confianza para asegurar el desarrollo del proyecto.

Además de los tres proyectos de la FAO, se elige para este estudio el proyecto del PNUD "Construyendo Soluciones Sostenibles". Este proyecto es el resultado de la cooperación entre PNUD, ACNUR y el Gobierno colombiano. Su objetivo principal es fortalecer a las comunidades priorizadas y las autoridades locales y nacionales en la transición hacia soluciones para la población desplazada en las tres fases del proceso: retorno, reubicación e integración local urbana. Gracias a la entrevista obtenida el día 14 de junio de 2019 con la señora Sandra Alvarado, una de 
las responsables del programa "Desarrollo Económico Incluyente Urbano" del área de reducción de la pobreza del PNUD, ha sido posible analizar el rol de la organización, los problemas principales encontrados y las dificultades en el cooperar con las comunidades locales y las instituciones nacionales.

El proyecto nace en 2012 y se desarrolla en 17 localidades y 10 departamentos del país. El programa se basa en un estudio de los documentos y evaluaciones de experiencias anteriores, con el fin de encontrar soluciones desde la base comunitaria. Antes del efectivo desarrollo del proyecto, se han lanzado algunos proyectos piloto, necesarios para disminuir el riesgo de fracaso, comprender el contexto y su complejidad. Además, se diseña un papel más central dentro del proyecto para el Gobierno y se favorece la interlocución y articulación con el nivel institucional y comunitario. Además, se garantiza la apertura del proyecto a diferentes actores y socios como la FAO, PMA, UE (United Nations Development Programme, n.d.).

En el caso específico de Altos de Florida el proyecto se desarrolla en tres puntos:

1. Mejora de la calidad de vida:

a. Apoyo y reconocimiento del derecho de parcelas a 500 familias.

b. Construcción o restauración de viviendas para 500 familias con el uso de empleo local.

c. Acceso a servicios básicos. Legalización y mejoras de las redes de agua potable y electricidad.

d. Desarrollo económico y social a través de un programa de microcréditos.

2. Fortalecimiento organizacional e institucional y fortalecimiento de la comunidad:

a. Apoyo a los desplazados internos, mujeres, pueblos indígenas y organizaciones juveniles en el diálogo con las autoridades locales.

b. Asistencia técnica para el diseño y establecimiento de los comités directivos locales.

c. Desarrollo de capacidades y capacitación sobre la planificación participativa para la prestación de servicios públicos. 
3. Protección y derechos de las víctimas: garantizar la protección mediante la presencia a través de la protección regular del personal de la ONU en la comunidad y coordinación con actores locales, apoyar la implementación de la Ley de Víctimas y Restitución de Tierras en el municipio, beneficiando a toda la comunidad (Ryan, 2012).

Adicionalmente, el PNUD contribuye técnica y financieramente al Centro de Atención y Orientación a Víctimas de Soacha (UAO); busca el fortalecimiento institucional en formación y capacitación, junto con la formulación de políticas públicas y la dotación de elementos físicos necesarios para su funcionamiento. La organización fortalece también el trabajo de un Centro de Empleo y Emprendimiento CEMPRENDE, en Soacha, que tiene como objetivo establecer una ventanilla única de emprendimiento, empleabilidad y microcrédito para población vulnerable/víctima (United Nations Development Programme, 2016).

Los últimos dos proyectos elegidos son "Familias en Acción' y "Jóvenes en Acción" de la Alcaldía de Bogotá y Soacha. A diferencia de los cuatro proyectos ya analizados que están concluidos, estos últimos dos continúan en marcha. Ambos se basan sobre un programa de transferencia condicional de dinero a familias consideradas vulnerables y en condiciones de extrema pobreza, y de ayudas económicas a los niños y jóvenes para los gastos en educación y salud.

El 13 de junio de 2019 tiene lugar la entrevista con los trabajadores en los proyectos y con el coordinador del proyecto "Familias en Acción", el señor Luis Francisco: el contenido de las entrevistas permite entender aún más en profundidad la complejidad del contexto de Soacha, afectado por graves emergencias de seguridad humana en algunas áreas. A esto hay que añadir los problemas relacionados con la no correspondencia de los datos del censo nacional con los datos reales y las crecientes dificultades para el personal extranjero a la hora de trabajar en esta zona.

Como afirma el Banco Interamericano de Desarrollo (2016), Familias en Acción es un programa de transferencias monetarias condicionadas que opera desde el año 2000 en Colombia. La población objetivo del programa son las familias pobres, desplazadas por el conflicto, comunidades indígenas que tienen integrantes menores de 18 años. Para identificar a los beneficiarios, 
se utiliza el SISBEN para medir el estándar de vida, así como los registros oficiales de las familias desplazadas por el conflicto, las comunidades indígenas y los beneficiarios de la Red Unidos.

A través de las entrevistas con el personal, ha sido posible entender la estructura en dos partes del proyecto:

a. Incentivo de salud: el programa ofrece ayudas económicas a las familias que satisfacen los requisitos necesarios, con niños de 0 a 6 años, en la compra de medicinas generales y otros artículos necesarios para la salud de los niños.

b. Incentivo a la educación: el programa ofrece ayudas económicas a las familias que satisfacen los requisitos necesarios, con niños de 4 a 18 años. Estas ayudas son destinadas a la compra de material escolar y para pagar las inscripciones a las escuelas.

El 13 de junio de 2019 tiene lugar la entrevista con el señor Francisco Alhucema Jaimes, actualmente coordinador del Programa de Discapacidad del Municipio de Soacha, anteriormente coordinador del programa Familias en Acción en la Comuna. El 11 de junio he tenido la oportunidad de entrevistar a la señora Olga Rocío Niño García, coordinadora del programa Prosperidad Social. Gracias a estas entrevistas, el estudio se enriquece de informaciones sobre el rol clave de las "madres líderes", sobre los problemas principales del proyecto y sobre su posible evolución. Las madres líderes son una figura fundamental para la consecución de una alta tasa de participación de la población en los proyectos, para una buena comunicación entre los participantes, y entre ellos y las instituciones. En suma, las madres líderes adquieren un papel fundamental para conseguir la eficacia del proyecto.

A modo de conclusión, y antes de explicar la metodología sobre la que se basa el trabajo de investigación cualitativa de campo realizada en Soacha, cabe destacar que los seis proyectos elegidos para este estudio representan una buena práctica a repetir en el futuro para mejorar la seguridad humana de la población más vulnerable de la comuna. La coordinación entre múltiples actores de varios niveles, de lo local a lo internacional, permite juntar esfuerzos hacia una misma dirección, construyendo sinergias y círculos virtuosos que afectan positivamente las 
condiciones materiales, humanas y sociales de las comunidades destinatarias de los proyectos. Como se dirá más adelante, los objetivos de seguridad humana no se consiguen a corto o a medio plazo. Es necesario adoptar una perspectiva a largo plazo que permita eliminar las causas subyacentes de las amenazas, riesgos y desafíos para la seguridad humana multidimensional de las personas y de las comunidades humanas. Esta afirmación debe tenerse aún más en cuenta cuando se implementan proyectos en contextos complejos y difíciles, como el de Soacha.

\section{Metodología}

El objetivo de estudio es el análisis del impacto de algunos proyectos de la FAO y PNUD sobre la percepción de la población sobre la seguridad humana, como ya ha sido explicado en el resumen. Con este objetivo se formula la siguiente pregunta de investigación: ¿cuál es el impacto de los proyectos de la red de las Naciones Unidas en la percepción de la población de Soacha sobre la seguridad? El análisis de esta pregunta ha sido estructurado a través de encuestas de carácter cualitativo. La encuesta consiste en ocho preguntas: las primeras tres son de carácter sociodemográfico, destinadas a dividir a los encuestados por sexo, edad y origen. Las restantes preguntas analizan la seguridad en el municipio, determinando si ha mejorado y cuánto: intentan determinar las dimensiones de la seguridad humana que - de acuerdo con las percepciones ciudadanas- han mejorado más, los problemas que siguen siendo más relevantes en Soacha y la valoración ciudadana de la actuación estatal para la seguridad humana. En relación con la pregunta número 7, esa ha sido estructurada tomando en cuenta los datos de la "Encuesta de Percepción y Victimización" realizada por la Cámara de Comercio de Bogotá en el año 2017, sobre todo para identificar las categorías de crímenes que siguen siendo las principales en el municipio (Chamber of Commerce of Bogota, 2018).

El cuestionario se entrega en formato papel, dejando a cada entrevistado la posibilidad de responder autónomamente y de forma anónima. En todo momento, en calidad de investigador responsable del estudio, he asegurado mi disponibilidad continuada para aclarar cualquier duda. Además, cada cuestionario comienza con una breve introducción del trabajo y de sus objetivos, y con una breve presentación personal del investigador. La muestra es de 120 habitantes, respetando las informaciones 
sobre edad y sexo de la población obtenidas a través de los datos oficiales gubernamentales. Esto, combinado con el hecho de que los participantes han sido reclutados en ambientes diversos (colegios, trabajo, calles) asegura la fiabilidad y la representatividad de la muestra. Durante las entrevistas personales, se ha detectado un alto grado de cierre hacia las instituciones y el personal extranjero por parte de las comunidades habitantes del territorio.

El formato breve del cuestionario responde esencialmente a una razón: un número elevado de preguntas podría disminuir el interés y la voluntad de los encuestados de responder correctamente y con sinceridad, especialmente los sujetos jóvenes y aquellos con un bajo nivel educativo. En caso de los encuestados pertenecientes a estas dos categorías, he garantizado siempre mi presencia para aclarar cualquier duda. Además, la comunidad local me ha acompañado para presentarme a estas categorías de entrevistados y poder explicarles los objetivos de la investigación.

El primer resultado destacable de las entrevistas obtenidas en Bogotá y Soacha es que uno de los principales problemas de la zona estudiada es la enorme diferencia entre los datos sobre el censo publicado por el Departamento Administrativo Nacional de Estadística (DANE) y las informaciones conseguidas en este estudio gracias al trabajo de campo. De hecho, el DANE en su última publicación de 2018 sobre la población de Soacha indica que en la comuna viven 634660 habitantes. Las entrevistas con el personal involucrado en los proyectos locales y los funcionarios pertenecientes a la Administración Pública llevan a concluir que en Soacha viven alrededor de un millón y medio de personas. Esta incongruencia, que ha llevado al Municipio a pedir oficialmente una revisión de los datos (El Tiempo, 2019), puede en parte explicarse por la incesante llegada de inmigrantes venezolanos y de desplazados internos, obligados a dejar sus tierras debido a las incursiones de grupos armados. La situación provoca la construcción de viviendas no autorizadas que, en poco tiempo, acaban convirtiéndose en barrios enteros, no reconocidos por las autoridades. El aumento poblacional vivido por Colombia provoca un fuerte crecimiento de los precios de las viviendas en las grandes ciudades, con Bogotá encabezando la lista, obligando a muchas familias a desplazarse hacia Soacha u otras áreas suburbanas, causando su gentrificación. Esto es porque, debido a su posición colindante con Bogotá, Soacha representa una opción cómoda y más asequible para muchos estudiantes y trabajadores (Flórez, 2017). 


\section{Características sociodemográficas}

Los resultados de las primeras tres preguntas, dirigidas a definir sexo, edad y origen de los encuestados, son conformes con los datos poblacionales ofrecidos por el DANE, antes mencionados. Entre las 110 personas entrevistadas hay 66 mujeres y 54 varones, reflejando parcialmente la división poblacional oficial según la cual el $51 \%$ de los habitantes registrados en Soacha son mujeres (Sistema de Estadística Territorial, 2019). Por lo que concierne a la edad, hay tres categorías establecidas: los jóvenes entre 10 y 25 años, los adultos entre 26 y 59 años y los mayores de 60 años. En cuanto al origen, los entrevistados tienen tres opciones: Soacha, otro municipio colombiano u otro país.

En cuanto a la segunda pregunta, el 63,33\% de los individuos declara tener una edad comprendida entre los 10 y los 25 años. El 25,83 \% son adultos y el 10,83 \% son mayores. Con respecto al origen, el 79,17\% de los entrevistados declara ser originario de Soacha y el $20,83 \%$ de otro municipio colombiano. No hay extranjeros entre los encuestados.

\section{La percepción poblacional sobre la seguridad}

La pregunta 4 pide a los encuestados clasificar la situación de la seguridad en el municipio de Soacha en los últimos cinco años. El $40 \%$ percibe el mismo nivel de seguridad, el 33,3\% un mayor nivel de seguridad, el 24,17 \% menor y el 2,4\% afirma no poder contestar. Un dato importante de destacar es que más del $50 \%$ de los jóvenes afirma que el nivel de seguridad percibido sigue siendo igual o se encuentra en disminución.

En caso de que la respuesta a la pregunta 4 sea "mejor", el cuestionario sigue con la pregunta 5, para determinar en qué medida la seguridad ha aumentado en las percepciones de los entrevistados. La pregunta 5 se presenta a 40 de ellos, entre los cuales 16 responden que la seguridad en los últimos cinco años ha aumentado "poco" (13,3\%), 14 contestan "mucho" (11,67\%), seis responden "bastante" (5 \%) y cuatro afirman "no saber" (3,30 \%).

En la pregunta número 6 , dirigida a evaluar cuáles dimensiones de la seguridad humana registran las mejoras más evidentes, el 49,17\% de los encuestados elige la seguridad 
ciudadana, el 15,83 \% la seguridad del tránsito, el 9,17\% la seguridad alimentaria, el 8,3 \% la seguridad sanitaria y el 17,5 $\%$ opta por no responder.

Cabe subrayar que nadie menciona la seguridad económica en su respuesta. Este factor podría corroborar que aspectos como la extrema pobreza y el desempleo siguen siendo percibidos como problemas urgentes que afectan a la comunidad y que aún no encuentran soluciones eficaces y duraderas. Esto a pesar de los muchos proyectos activos y destinados a eliminar la inseguridad alimentaria para las familias de Soacha.

La pregunta 7 profundiza en las categorías de crímenes que, según la percepción de los entrevistados, afectan mayormente a la seguridad ciudadana en Soacha: hurto, venta de drogas, homicidio común, robo, acoso sexual y, por último, lesiones y otros daños físicos. El 42,5\% afirma estar preocupado por los hurtos, el 37,5 \% hace referencia a la venta de drogas, el 7,5 $\%$ a las lesiones y a otros daños físicos. Para terminar, el 2,5 $\%$ contesta el robo y el 10,8 \% no contesta. En relación con la dimensión de la seguridad humana relacionada con la (in) seguridad ciudadana, el alto nivel de crímenes ha provocado la toma de algunas decisiones muy contundentes por parte de las autoridades para poner freno al fenómeno de la delincuencia común. Como confirmación de esto, durante las entrevistas realizadas en Soacha, varias personas refieren que en algunos barrios del municipio se impone un toque de queda a partir de ciertas horas de la noche con el objetivo de llevar a cabo lo que definen como una operación generalizada de "limpieza social".

Otro aspecto relevante es que el homicidio común, el secuestro y las amenazas directas (estos últimos dos están incluidos en la categoría "otros") no aparecen en ninguna de las respuestas de los 110 cuestionarios entregados a la población. Esto está en desacorde con la información recogida durante las entrevistas personales con las autoridades y los trabajadores de los proyectos en Soacha.

La última pregunta, destinada a determinar la percepción y evaluación de la actuación estatal por parte de la ciudadanía de Soacha, ofrece siete opciones como respuesta: 1 (pésima), 2 (muy mala), 3 (mala), 4 (menos que regular), 5 (aceptable), 6 (muy buena), 7 (excelente), 8 (no sabe o no responde). 
El 27,50 \% evalúa el resultado de la acción estatal como malo, el 26,7 \% menos que regular, el 17,55 \% aceptable, el 13,3\% muy malo, el 7,5 \% pésimo, el 4,2 \% muy bueno, el 3,3 \% no sabe o prefiere no responder. Nadie elige excelente.

\section{Reflexiones sobre los resultados obtenidos a través de la encuesta}

Los datos relativos a la evaluación de la actuación del Estado para la seguridad en el municipio de Soacha (pregunta 8 ) tienen mucho en común con cuanto emerge del informe de 2018 del Latinobarómetro, que muestra el nivel de confianza ciudadana en las instituciones de Colombia. El documento indica que la confianza en el poder judicial se sitúa en el $23 \%$, la confianza en el Gobierno nacional llega al $22 \%$ y en los partidos políticos al $16 \%$ (Corporación Latinobarómetro, 2018).

Estos factores son conformes con los resultados del presente estudio, especialmente en relación con las dificultades detectadas en los intentos de desarrollar nuevos proyectos en Soacha: la población ha perdido confianza hacia las instituciones públicas por la reiterada ausencia del Estado en el territorio y esto provoca su escasa involucración y participación en los nuevos proyectos y actuaciones, especialmente si provienen del sector público.

La confianza en las instituciones es, a largo plazo, un factor determinante para el éxito de las políticas públicas. En muchos casos, las reformas implican sacrificar el apoyo ciudadano a corto plazo en favor de ganancias a largo plazo. En un contexto de alta confianza ciudadana, las reformas operadas por el Estado pueden aprobarse e implementarse rápida y adecuadamente, pudiendo llegar a mantenerse por un plazo suficiente para dar resultados visibles a la opinión pública. En un clima de baja confianza, los ciudadanos tienden a valorar más los resultados inmediatos, que aportan generalmente beneficios parciales: esto induce a los políticos a buscar ganancias a corto plazo, incapaces de resolver problemas estructurales como las causas profundas de la pobreza extrema y las desigualdades (Organization for Economic Cooperation and Development, 2013).

En consonancia con lo dicho anteriormente, cabe destacar que la FAO y otras organizaciones internacionales juntan esfuerzos para resolver las amenazas y retos a la seguridad alimentaria de muchas familias en el territorio, sin conseguir 
los resultados esperados. Cabe recordar que, en la pregunta 6 , apenas el 8,3 \% expresa que la dimensión sanitaria es la que más ha mejorado en Soacha, y sólo el 9,17 \% contesta mencionando la dimensión alimentaria. Además, nadie considera que la seguridad económica es la que registra los mejores datos, dejando implícito que los problemas como el desempleo y la pobreza persisten en el territorio de manera generalizada.

El parcial fracaso de los esfuerzos de las organizaciones internacionales en Soacha depende de muchos factores. Los resultados de las entrevistas realizadas con el personal de la FAO en Bogotá permiten destacar los problemas relacionados con la difícil continuidad y coherencia de los proyectos en contextos que cambian rápidamente debido a los fenómenos de gentrificación de las áreas periféricas del Distrito Capital, mencionados anteriormente. A esto hay que sumar la citada escasa confianza en las instituciones y en su personal, lo que dificulta la cooperación de la población. Los bajos niveles educativos por parte de la ciudadanía impiden poner en práctica los conocimientos transmitidos sobre cómo cocinar, manipular los alimentos y recoger agua potable.

Otro dato interesante radica en la comparación entre los resultados de las preguntas 6 y 7 . La primera destaca que la seguridad comunitaria es, para la percepción ciudadana, la dimensión que más mejoras registra en Soacha en los últimos cinco años. La segunda pregunta registra que la población percibe el hurto y la venta de drogas como las principales amenazas para su seguridad en este ámbito. Contra estas amenazas no hay una coordinación en la actuación multinivel requerida para garantizar la seguridad humana a largo plazo, que busca crear sinergias entre las autoridades locales, estatales y los trabajadores de las organizaciones internacionales con proyectos activos en el territorio.

\section{Conclusiones}

El Municipio de Soacha se caracteriza por tener una elevada concentración de problemas de diferente naturaleza: sociales, económicos, medioambientales, demográficos, alimentarios, educativos, junto con amenazas para la seguridad personal y para la igualdad de género. La incongruencia entre los datos oficiales del DANE y las estimaciones basadas en el trabajo de campo sobre la población actual conlleva importantes consecuencias. Entre ellas, una cantidad de fondos estatales inferior a la cantidad 
que el municipio necesita de acuerdo con su tamaño real, así como un número de estructuras y servicios inadecuados a las reales necesidades de la población.

Además, la proliferación de viviendas ilegales provoca evidentes desequilibrios para la seguridad comunitaria: el 48 $\%$ de los 378 barrios y urbanizaciones de Soacha son ilegales, es decir que 152 de ellos se encuentran en situación de asentamiento (PROBOGOTÁ, n.d.). La ilegalidad de las viviendas acarrea graves consecuencias directas para sus habitantes: la falta de electricidad, agua potable y otros suministros básicos, la inexistencia de una red de alcantarillas y la imposibilidad de acceso al transporte público. Estas carencias influencian negativamente la calidad de vida de las personas y tienen repercusiones directas sobre la economía familiar, empezando por la falta de alimentos básicos en la que se centran los proyectos de la FAO.

Además, en estos barrios, numerosas organizaciones criminales sientan sus bases estratégicas: algunas de ellas están en estrecho contacto con los carteles del narcotráfico, como el Cartel de Sinaloa o el Cartel del Golfo (Ocampo Rodríguez, 2019). Estas áreas están separadas por confines invisibles que dibujan equilibrios frágiles y en constante tensión. Muchos de estos grupos armados y organizaciones criminales se financian mediante la venta de droga, uno de los problemas más relevantes del Municipio que afecta a numerosos jóvenes. En efecto, está creciendo drásticamente el consumo de droga en los jóvenes de 13 y 14 años, que cada vez más frecuentemente recurren al atraco y al robo, incluso armado, para poderla comprar (Periodismo Público, 2016). Este fenómeno representa para muchos uno de los problemas principales porque los jóvenes menores de 25 años representan el 44,35 \% de la población total (Sistema de Estadística Territorial, 2019).

Uno de los factores que más caracterizan el contexto de Soacha es la ingente presencia de desplazados nacionales, caracterizados por tener niveles de vulnerabilidad y dependencia más altos que los pobres y los indigentes urbanos. Esta tendencia al aumento se debe también a las difíciles condiciones económicas de los colectivos mayoritarios de la población y a la elevada tasa de desempleo (Ibáñez y Moya, 2007). Como se ha explicado antes, el desplazamiento forzado no es algo nuevo en la historia de Colombia. De hecho, es un fenómeno complejo y 
de carácter casi permanente debido a las múltiples dinámicas sociales, económicas y políticas que lo vieron provocado (Ruscheinsky, Baltazar, 2013).

El problema más grande encontrado por las organizaciones que desarrollan proyectos e implementan políticas de inclusión de la población desplazada es la construcción de una relación de confianza con los destinatarios de los proyectos y de las políticas. En distintos casos ha sido necesaria la ayuda de intermediarios y la intervención de funcionarios públicos para consentir el acceso del personal a la comunidad.

Personas como las "Madres líderes" han sido esenciales, no sólo para permitir la entrada de las organizaciones al territorio donde viven las comunidades, sino también para garantizar el buen funcionamiento de los proyectos. Las "Madres líderes" han asegurado la participación de los ciudadanos, han velado por la seguridad del personal trabajador de los proyectos y han permitido la comunicación y el diálogo entre la comunidad y la organización de los proyectos. Este factor ha sido, y seguirá siendo, fundamental para la implementación de futuros proyectos.

Uslaner y Glaesner describen el precio que pagan las comunidades a causa de la corrupción como una "trampa de desigualdad": los países con un elevado nivel de percepción de la corrupción son también aquellos con el nivel más alto de desigualdad (Uribe, 2019). Este coste negativo también se refleja en el desarrollo y en la continuación de los proyectos, como explicado en una entrevista en las Naciones Unidas y luego confirmado en las entrevistas sucesivas realizadas en Soacha. Como se menciona anteriormente, uno de los problemas más importantes es la difícil relación entre la población y las instituciones estatales y los escasos apoyos ofrecidos por éstas a la comunidad.

La incertidumbre política no permite la fácil creación de planes sociales en algunas áreas de Colombia, sobre todo si el Estado no puede garantizar la seguridad del personal en el campo y deja a las organizaciones la difícil tarea de construir desde cero las relaciones con las comunidades que viven en contextos complejos y peligrosos. 
A pesar de poder evaluar positivamente los resultados iniciales de muchos proyectos, surge el riesgo de que, en el largo plazo, los efectos secundarios podrían empezar a afectar negativamente a algunos segmentos de la comunidad. Los proyectos con más recursos prevén trasferencias directas de dinero a favor de las familias vulnerables y con ingresos bajos para fortalecer su educación sobre la seguridad alimentaria y sanitaria, para consolidar el rol de las mujeres en el contexto familiar y comunitario, y para asegurar el abastecimiento de los suministros básicos como agua o electricidad.

Como se ha dicho anteriormente, la necesidad de mostrar resultados a corto plazo para asegurarse fondos en los años futuros y la desconfianza en las instituciones nacionales obstaculizan el desarrollo de una cooperación constructiva entre organizaciones extranjeras y nacionales, dejando a estas últimas la obligación de continuar sin medios suficientes los proyectos desarrollados, que acaban siendo suspendidos. Además de crear desequilibrios intracomunitarios, esta situación puede favorecer la consolidación de costumbres difíciles de eliminar, como la dependencia de las familias hacia la ayuda monetaria directa proveniente del proyecto. Esta dependencia impide que las familias sean capaces de sobrevivir sin las ayudas y que no tengan ningún estímulo o incentivo para desarrollar nuevas capacidades para mejorar su calidad de vida.

El análisis de los resultados de las entrevistas muestra que la percepción de la población es que la situación general sobre la seguridad en Soacha no ha vivido cambios sustanciales durante los últimos cinco años. A pesar de poder tener una interpretación negativa en el corto plazo, hay que proyectar este dato en el largo plazo. Desde el comienzo de la implementación de los proyectos relacionados con la seguridad humana, en Soacha hay más familias y jóvenes que reciben ayuda y asistencia, registrando una mejor calidad de vida. Además, hay cada vez familias e individuos que solicitan asistencia. Para poner un ejemplo, el proyecto para la Alimentación y la Agricultura, apoyado por la FAO, se ha dado por terminado una vez que el personal ha verificado la autosuficiencia de la comunidad interesada.

Adoptando una perspectiva a largo plazo, de acuerdo con el enfoque de la ONU sobre seguridad humana, podemos registrar un fenómeno que, por muy pequeño que sea, representa un cambio positivo hacia el empoderamiento de las comunidades locales que viven en Soacha. Este es el camino que hay que seguir, con el apoyo de las organizaciones pertenecientes a la red 
ONU, sobre todo la FAO y el PNUD, las instituciones nacionales, locales y otros actores de la sociedad civil, como las ONG y otras asociaciones ciudadanas.

El proceso de construcción de la confianza requiere tiempo, sobre todo si se debe dar en un contexto caracterizado por una fuerte presencia de inmigrantes, desplazados, comunidades vulnerables, comunidades indígenas y familias que viven en condiciones de extrema pobreza. Este proceso no se puede acelerar o forzar y se debe reconocer su fragilidad y equilibrio dinámico y precario, siendo continuamente amenazado por organizaciones criminales, grupos armados, la corrupción o factores externos.

Soacha representa uno de los retos principales no solo para Colombia o América del Sur, sino también para la comunidad internacional. Se caracteriza por ser, en efecto, un "laboratorio y una experiencia social" emblemática: es una gran ciudad perteneciente al área metropolitana de una capital estratégica para América del Centro-Sur, con unos sesgos sociales comunes a muchas áreas periféricas de las grandes ciudades americanas: extrema pobreza, fuertes desigualdades, el riesgo para los jóvenes de tener un futuro sin posibilidades ni expectativas y la amenaza del crimen organizado, entre otros.

A esto hay que sumar los factores globales externos que pueden afectar a estas áreas de manera más contundente si se compara con los barrios ricos de Bogotá y de las otras metrópolis centro y suramericanas. Piénsese a la guerra de aranceles y al giro proteccionista en la política comercial estadounidense, lanzados por el presidente Donald Trump, o, aún más preocupante, a la crisis global desencadenada por la COVID-19.

A modo de conclusión, el esfuerzo hecho hasta ahora en Soacha es un factor de cambio positivo, pero aún insuficiente. Para garantizar mejoras duraderas para la seguridad humana en el municipio, que sean estructurales y a largo plazo, es esencial la aportación de la sociedad colombiana en general, de la sociedad bogotana en especial, y -sobre todo- del Gobierno estatal, junto con el apoyo humano y financiero de la comunidad internacional, coordinado por los organismos pertenecientes a la red de las Naciones Unidas. Una 
intervención sinérgica, multinivel y coordinada en el largo plazo es la única que puede promover la seguridad humana en Soacha sin interferir en los frágiles equilibrios existentes.

\section{Bibliografía}

Chamber of Commerce of Bogota. (2005). Estado de Bogotá Región. Bogotá: Cámara de Comercio de Bogotá.

Chamber of Commerce of Bogota. (2012). Observatorio de la seguridad en Bogotá: Balance del 2011 N. ${ }^{\circ} 42$. Bogotá: Centro de Información Empresarial.

Chamber of Commerce of Bogota. (2018). Encuesta de percepción y victimización en Soacha, 2017. Bogotá: Vicepresidencia de Articulación Público - Privada Dirección de seguridad ciudadana y empresarial.

Castellanos, R. (1991). Soacha un Laboratorio de Problemas. El Tiempo. Retrieved from https://www.eltiempo.com/archivo/documento/MAM-146527

Corporación Latinobarómetro. (2018). Informe 2018. Santiago de Chile. Banco de datos en línea.

Dávila, J. (2013). Urban mobility and poverty: lessons from Medellín and Soacha, Colombia. Medellín: Development Planning Unit, UCL \& Faculty of Architecture, Universidad Nacional de Colombia.

El Tiempo. (1999). Soacha Refugio de la Pobreza. El Tiempo. Recuperado de https:// www.eltiempo.com/archivo/documento/MAM-940317

El Tiempo. (2019). Soacha pide al DANE revisar las cifras del censo nacional 2018. El Tiempo. Recuperado de https://www.eltiempo.com/bogota/soacha-pide-al-dane-revisar-las-cifras-del-censo-nacional-2018-319828\# : : text $=$ Soacha $\% 20$ pide $\% 20$ al\%20DANE\%20revisar\%20las $\% 20$ cifras $\% 20$ del\%20Censo $\% 20$ Nacional,de\%20poblaci\%C3\%B3n\%20en\%20Soacha\%2C\%20Cundinamarca.\&tex$\mathrm{t}=$ Encuentra\%20la\%20validaci\%C3\%B3n\%20de\%20El\%20Cazamentiras $\% 20$ al\%20final\%20de\%20la\%20noticia.

Flórez, J. (2017). Soacha: el karma de crecer a la sombra de un gigante. Semana. Recuperado de https://www.semana.com/nacion/articulo/soacha-historia-censo-y-crecimiento-poblacional/541529

Food and Agriculture Organization (FAO). (2015). Modelos técnicos pedagógicos para la producción de alimentos en huertas escolares, como una estrategia para la complementación de los menús en los comedores y el mejorameniento de la salud nutricional de los niños de Soacha/ resultados proyectos UTF/COL/058/COL. FAO.

Gallego Suárez, S. (2011). El 'desafío' de vivir en Altos de la Florida. Periodismo Público.

González, N., (2015), Las huertas escolares una estrategia para fortalecer la salud y la alimentación. Food and Agriculture Organization.

González, N., (2016), Comunidades educativas en Soacha comen sano y tienen mejor salud nutricional. Food and Agriculture Organization.

Ibáñez A., Moya A. (2007). La población desplazada en Colombia: examen de sus condiciones socioeconómicas y análisis de las políticas actuales, Departamento Nacional de Planeación. Bogotá. Departamento Nacional de Planeación.

Inter-American Development Bank (IABD), (2016). Síntesis del Programa más Familias en Acción en Colombia. IABD.

Inter-American Institute of Human Rights (IIDH), \& United Nations Development Programme (UNDP). (2012). Conferencia latinoamericana sobre seguridad humana y las agendas de integración regional. San José: IIDH UNDP.

Niño Pavajeau, J. (1999). Las migraciones forzadas de población, por la violencia, en Colombia: una historia de éxodos, miedo, terror, y pobreza. Scripta Nova Revista Electrónica de Geografía y Ciencias Sociales, 45(33).

Ocampo Rodríguez, A. (2016). LAfm. Clan del Golfo y carteles mexicanos estarían operando en Soacha. Office for the Coordination of Humanitarian Affairs. (2009). Human security in theory and practice. New York: United Nations. 
Organization for Economic Cooperation and Development (OECD). (2013). Government at a glance 2013, trust in government, policy effectiveness and governance agenda. OECD.

Periodismo Público. (2016). Se desborda consumo de drogas entre los jóvenes de Soacha. Periodismo Público. Recuperado de https://periodismopublico.com/se-desborda-consumo-de-drogas-entre-los-jovenes-de-soacha

PROBOGOTÁ. Conozca Soacha, uno de los principales municipios de Cundinamarca. Recuperado de http://probogota.org/municipios/soacha/ Último acceso: 6 de mayo de 2020.

Ryan, J., (2012). UNDP - UNCHR: transitional solutions initiative. From the assistance to sustainable solutions for IPDS. The United Nations Development Porgramme.

Rocío, O. \& García, R., (2007). Proyecto Seguridad Alimentaria Urbana Comuna 4 - Municipio de Soacha RESA. Food and Agriculture Organization.

Ruscheinsky, A., \& Baltazar, E. (2013). Los desplazamientos forzados como riesgos sociales asociados a las condiciones de la violencia política en Colombia. Sociologías, 15(34). DOI: 10.1590/s1517- 45222013000300008

Sistema de Estadística Territorial (2019). Soacha, Cundinamarca, TerriData, Bogotá.

United Nations Development Programme. Programa Construyendo Soluciones Sostenibles - TSI. United Nations

United Nations Development Programme. (1994). Human Development Report 1994. New York: Oxford University Press.

United Nations Development Programme. (2016). En Soacha nuevo centro regional de atención integral a víctimas. United Nations.

United Nations High Commissioner for Refugees. (2013). Operación del ACNUR en Soacha, Cundinamarca. United Nations

Uribe L., Isaza C., Franco D., García-Márquez P., Sarmiento L. (2019). Corruption trap: the case of Colombian regions, the organization for economic co-operation and development. OECD Global Anti- Corruption \& Integrity Forum. 